\title{
The influence of jet geometry on light curves and spectra of GRB afterglows
}

\author{
A. G. Tolstov ${ }^{1,2}$ \\ ${ }^{1}$ Institute for Theoretical and Experimental Physics (ITEP), Bolshaya Cheremushkinskaya 25, 117218 Moscow, Russia \\ e-mail: tolstov@mail.itep.ru \\ 2 Max Plank Institute for Astrophysics(MPA), Karl-Schwarzschild-Str. 1, 85741 Garching, Germany \\ e-mail: tolstov@mpa-garching.mpg.de
}

Received 27 May 2004 / Accepted 4 January 2005

\begin{abstract}
We have performed detailed calculations of spectra and light curves of GRB afterglows assuming that the observed GRBs can have a jet geometry. The calculations are based on an expanding relativistic shock GRB afterglow model where the afterglow is the result of synchrotron radiation of relativistic electrons with power-law energy distribution at the front of external shock being decelerated in a circumstellar medium. To determine the intensity on the radiation surface we numerically solve the full time-, angle- and frequency-dependent special relativistic transfer equation in the comoving frame using the method of long characteristics.
\end{abstract}

Key words. gamma rays: bursts - ISM: jets and outflows - radiative transfer

\section{Introduction}

At the present time we know that gamma-ray bursts (GRBs) are explosive phenomena at cosmological distances. If the emission is isotropic, estimations based on observations give us values of released energy up to $E_{0} \sim 3.4 \times 10^{54} \mathrm{erg}$ for GRB990123, that exceeds the rest energy of a solar mass star (Kulkarni et al. 1999). To reduce this large amount of energy it can be supposed that the GRB emission is highly collimated. Better evidence for jet structure is the achromatic break in light curves (Sari et al. 1999a) seen in many afterglows, e.g. GRB990123 (Kulkarni et al. 1999) and GRB990510 (Harrison et al. 1999; Stanek et al. 1999). Furthermore, spherical symmetry conflicts with the linear polarization (Sari 1999b) observed for a few afterglows (Covino et al. 1999; Wijers et al. 1999).

Generally, a GRB jet can display an angular structure and can be seen by an observer at a wide range of viewing angles from the jet axis (Wei \& Jin 2003; Granot \& Kumar 2003). Here, we consider a jet with uniform angular structure taking into account the effect of the equal-arrival-time surface at different angles of the observation and show what changes in the GRB afterglow are produced in the transition from spherical symmetry to jet geometry.

The evolution of the jet and the light curves has been widely investigated (Panaitescu \& Meszaros 1999; Kumar \& Panaitescu 2000), including lateral jet expansion (Salmonson 2003), investigation of the "structured" jet (Granot \& Kumar 2003), 3D numerical simulations of the jet dynamics
(Canizzo et al. 2003) and different angles of observation (Granot et al. 2002). In all these papers the transfer equation for the resulting light curves calculation has not been accurately solved and these works are based on simple expressions for local emissivity. They either focus on the power-law branch of the spectrum between the break frequencies, or some other simple assumptions about the characteristics of the radiation field.

In this paper we present a detailed calculation of spectra and light curves based on numerical solution of the special relativistic transfer equation in the comoving frame of reference. We will show that the exact calculation of intensity, depending on the angle to the surface normal, can have a critical influence on the form of the spectra visible to an observer. The exact determination of isochronous surfaces, which is important for the explanation of the observed luminosity in GRBs, and comprehension of their spectral properties (Bianco \& Ruffini 2004) are also taken into account in our calculations.

For the accurate calculation of intensity on the surface of a radiating structure, the transfer equation must be solved by integrating the emission through the structure and the following flux calculations. The calculation is based on the model where the jet is cut from a spherically symmetric flow. We add two parameters to take into account a radial jet structure and different values of observer viewing angles. In the next section we discuss our model in more detail, in Sect. 3 we calculate the emission for different values of the parameters and we present a discussion and some conclusions in Sect. 4. 


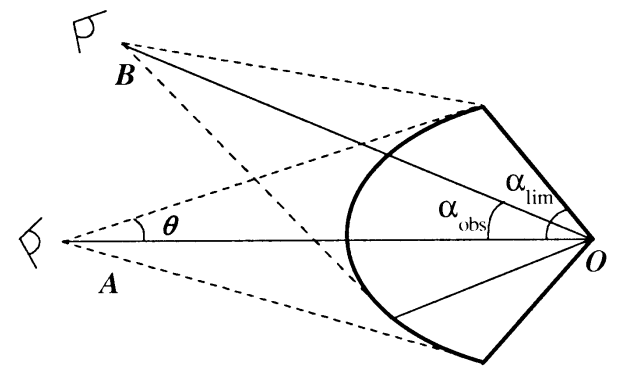

Fig. 1. The part of the quasi-ellipsoid from which the photons reach a remote observer. The explosion center is located at the vertex of the jet opening angle $\alpha_{\mathrm{lim}}$. The jet is observed under the angle $\alpha_{\mathrm{obs}}$ between the jet symmetry axis and the direction towards the observer.

\section{Physical model}

Our jet geometry investigation is based on the numerical solution of the problem in the spherically-symmetric case. To take into account jet geometry, first we fix the direction towards the observer in the case of spherical symmetry. Because of the high shock velocity, light at a certain time reaches the observer from an ellipsoidal structure (Fig. 1). To construct the jet, we cut the cone with its axis as the direction towards the observer $A$ and the angle $\alpha_{\text {lim }}$ forming jet opening angle. The line of sight of observer $A$ in this case coincides with the jet axis. To consider the jet under different values of the viewing angle, we add the angle $\alpha_{\text {obs }}$ between the observer $B$ and the jet axis. This approach gives us the possibility to consider some jet effects without using specific hydrodynamics code and more complicated transfer equations.

We briefly consider the model we have used for numerical calculations of spectra and light curves in the case of spherical symmetry (Tolstov \& Blinnikov 2003). A more detailed formal description is given by Granot \& Sari (2001).

In general, the transfer and hydrodynamic equations constitute a combined system of equations. In our problem, however, we solve them separately. To determine the variables of the medium we use a self-similar solution for a relativistic shock for an ultrarelativistic gas in the case of the conservation of total shell energy (Blandford \& McKee 1976). The solution describes the explosion with a fixed amount of energy $E_{0}$ and propagation of a relativistic shock through a uniform cold medium.

$\Pi=\frac{1}{3} e=\frac{2}{3} w_{0} \Gamma^{2} \chi^{-17 / 12} ; \quad \gamma^{2}=\frac{1}{2} \Gamma^{2} \chi^{-1} ; \quad n \gamma=2 n_{0} \Gamma^{2} \chi^{-7 / 4}$,

where

$\Gamma^{2} \propto t^{-3} ; \quad \chi=\left[1+8 \Gamma^{2}\right](1-r / t)$.

Above, $\Gamma$ is the Lorentz factor of the shock front, $\gamma, \Pi, e, n$ the Lorentz factor, pressure, energy and density of the shocked fluid, measured in the local rest frame of fluid, respectively, and $n_{0}, w_{0}$ is the density and enthalpy of the external medium.

For accurate calculation we should also know the electron energy spectrum and the magnetic field strength. Here for the local emissivity calculation we use the conventional assumptions on relativistic electrons (e.g. Sari et al. 1999c). Based on the standard fireball shock model we assume
(Zhang \& Meszaros 2003) that the electrons have a power-law distribution and that their total energy behind the shock front accounts for $\epsilon_{\mathrm{e}}$ of the internal energy:

$N\left(\gamma_{\mathrm{e}}\right)=K_{0} \gamma_{\mathrm{e}}^{-\mathrm{p}} ; \quad \gamma_{\mathrm{e}} \geq \gamma_{\min , 0}=\frac{\epsilon_{\mathrm{e}} e_{0}}{n_{0} m_{\mathrm{e}} c^{2}}$,

where $m_{\mathrm{e}}$ is the electron mass, $e_{0}$ is the energy density of the external medium and $K_{0}=(p-1) n_{0} \gamma_{\min , 0}^{\mathrm{p}-1}$.

As the electrons pass through the shock, they begin to lose energy due to adiabatic cooling determined by the solution of Blandford and McKee (1976) and due to radiative losses which can be calculated from synchrotron theory. These assumptions have an influence on the resulting electron power law energy distribution. According to Granot \& Sari (2001) we can use the distribution:

$N\left(\gamma_{\mathrm{e}}, \chi\right)=K_{0} \gamma_{\mathrm{e}}^{\mathrm{p}} \chi^{-\frac{7(\mathrm{p}+2)}{6}}\left(1-\frac{\gamma_{\mathrm{e}}}{\gamma_{\max }(\chi)}\right)$,

$\gamma_{\min }(\chi) \leq \gamma_{\mathrm{e}} \leq \gamma_{\min }(\chi)$

where $\gamma_{\min }(\chi)=\gamma_{\mathrm{e}}\left(\gamma_{\min , 0}, \chi\right)$, and $\gamma_{\max }(\chi)$ is the maximum Lorentz factor at $\chi>1$, which corresponds to an electron with $\gamma_{\mathrm{e}, 0} \rightarrow \infty$.

The magnetic field $B$ is parameterized by the quantity $\epsilon_{\mathrm{B}}$, which equals the fraction of the internal energy contained in the magnetic field $B^{2}=8 \pi \epsilon_{\mathrm{B}} e$. The magnetic field is randomly oriented and decreases with time due to adiabatic expansion of the shell.

Here we present only the basic formulae for synchrotron radiation used in our calculation. The synchrotron power per unit frequency $P(\omega)$ (for one electron) is:

$P(\omega)=\frac{3^{5 / 2}}{8 \pi} \frac{P_{\mathrm{sy}}}{\omega_{\mathrm{c}}} F\left(\frac{\omega}{\omega_{\mathrm{c}}}\right)$,

where

$P_{\mathrm{sy}}=\frac{1}{6 \pi} \sigma_{\mathrm{Th}} c B^{2}\left(\gamma_{\mathrm{e}}^{2}-1\right) ; \quad \omega_{\mathrm{c}}=\frac{3 \pi}{8} \frac{e B}{m_{\mathrm{e}} c} \gamma_{\mathrm{e}}^{2}$.

Here $P_{\text {sy }}$ is the frequency-integrated synchrotron power averaged over the pitch angle, $\sigma_{\mathrm{Th}}$ being the cross-section for electron scattering, $\gamma_{\mathrm{e}}$ the electron Lorentz factor, $e$ and $m_{\mathrm{e}}$ the electron charge and mass, $F(u)$ - a standard synchrotron radiation function (Rybicki \& Lightman 1979). The synchrotron absorption coefficient $\chi$ is specified by the formula:

$\chi=\frac{1}{8 \pi m_{\mathrm{e}} v^{2}} \int_{\gamma_{\mathrm{e}, \text { min }}}^{\gamma_{\mathrm{e}, \text { max }}} \mathrm{d} \gamma_{\mathrm{e}} \frac{N\left(\gamma_{\mathrm{e}}\right)}{\gamma_{\mathrm{e}}^{2}} \frac{\mathrm{d}}{\mathrm{d} \gamma_{\mathrm{e}}}\left(\gamma_{\mathrm{e}}^{2} P\left(\omega, \gamma_{\mathrm{e}}\right)\right)$.

To determine the intensity on the radiation surface we numerically solve the full time-, angle- and frequency-dependent relativistic transfer equation in the comoving frame of reference using the method of long characteristics up to the values of the Lorentz factor $\gamma \sim 1000$.

After the calculation of intensity the flux can be determined:

$F_{0, \mathrm{t}_{\mathrm{obs}}}=\frac{2 \pi}{D^{2}} \int_{\mu_{0, \min }}^{1} \mu_{0} R^{2} I\left(r\left(\mu_{0}\right), v_{0}\left(\frac{v}{v_{0}}\right), \cos \delta\left(\cos \delta_{0}\right)\right)\left(\frac{v_{0}}{v}\right)^{3} \mathrm{~d} \mu_{0}$, 


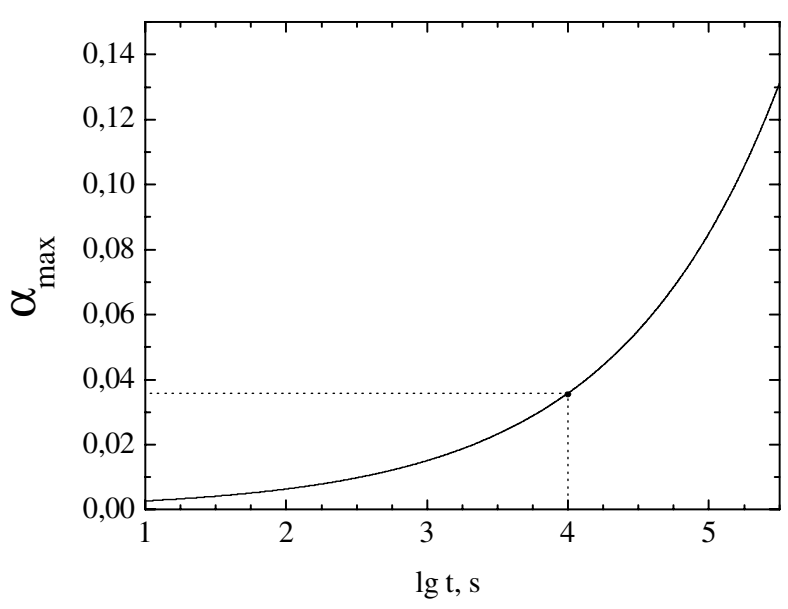

Fig. 2. The angle visible to the observer from an emitting structure increases with time. Time is measured in the observer's frame of reference. For $t=10^{4} \mathrm{~s}$ it is shown that there is no reason to increase the jet opening angle more that 0.037 .

where subscript 0 is related to the observer frame, $D$ is the distance to the observer, $R$ is the radius of the shell and $\mu_{\min }$ is the cosine of the maximum angle visible to the observer.

Thus, the observed afterglow spectra and light curves depend on the hydrodynamic evolution, the radiation processes, the distance to an observer and two parameters we have used to take into account the jet structure: the jet opening angle $\alpha_{\lim }$ and the viewing angle $\alpha_{\text {obs }}$.

To solve the problem in the case of spherical symmetry we have used the following parameters (Tolstov \& Blinnikov 2003): $E_{0}=10^{53} \mathrm{erg}, n_{0}=1 \mathrm{~cm}^{-3}, \epsilon_{\mathrm{e}}=0.5, \epsilon_{\mathrm{B}}=0.1, p=2.5$, $D=10^{27} \mathrm{~cm}$. In the next section we consider the influence of the jet geometry on spectra and light curves by varying $\alpha_{\text {lim }}$ and $\alpha_{\text {obs. }}$.

\section{Results of the numerical calculation}

As the shell from which the light reaches an observer moves towards the observer, the visible angle of the structure increases with time. This dependence is presented in Fig. 2, where $\alpha$ is the angle between the direction to the observer and the line that connects the center of the symmetry with a point on the surface that is still visible to the observer. There is the following relationship between the real observation angle $\theta$ and $\alpha$ :

$\cos \theta=\frac{1-\mu \rho(\mu)}{\left[1+\rho^{2}(\mu)-2 \rho(\mu) \mu\right]^{1 / 2}}$,

where $\mu=\cos \alpha, \rho=R\left(t_{\mathrm{obs}}\right) / D, t_{\mathrm{obs}}=t_{\mathrm{obs}}(\mu, \rho, D), D$ is the distance from the center of the burst to the observer, and time in the burst frame of reference $t$ is connected to the time in the observer's frame of reference $t_{\mathrm{obs}}$ by the formula:

$$
\begin{aligned}
t_{\mathrm{obs}} & =t+\frac{D\left(1-\left[1+\rho^{2}(\mu)-2 \rho(\mu) \mu\right]^{1 / 2}\right)+R_{0}}{c} \\
& \approx t+\frac{R_{0}-R\left(t_{\mathrm{obs}}\right)}{c} \quad(\rho \ll 1)
\end{aligned}
$$

if we suppose that the initial time of the observation corresponds to the initial time of the burst $\left(t_{0}=t_{0, \mathrm{obs}}+D / c\right)$ and that initial radius of the burst structure is $R_{0}$.

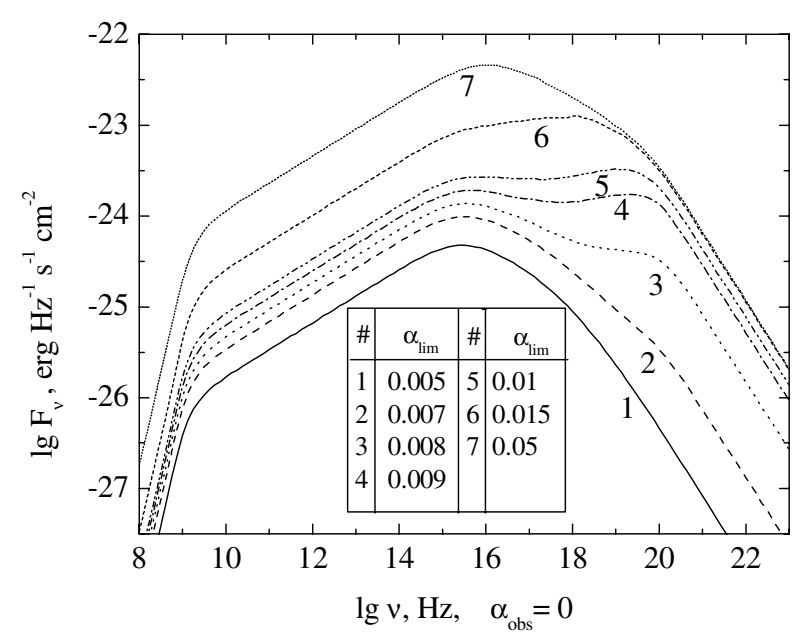

Fig. 3. Instantaneous afterglow spectra at time $t=10^{4} \mathrm{~s}$ and the observational angle $\alpha_{\mathrm{obs}}=0$ for different values of the limiting angle $\alpha_{\mathrm{lim}}$.

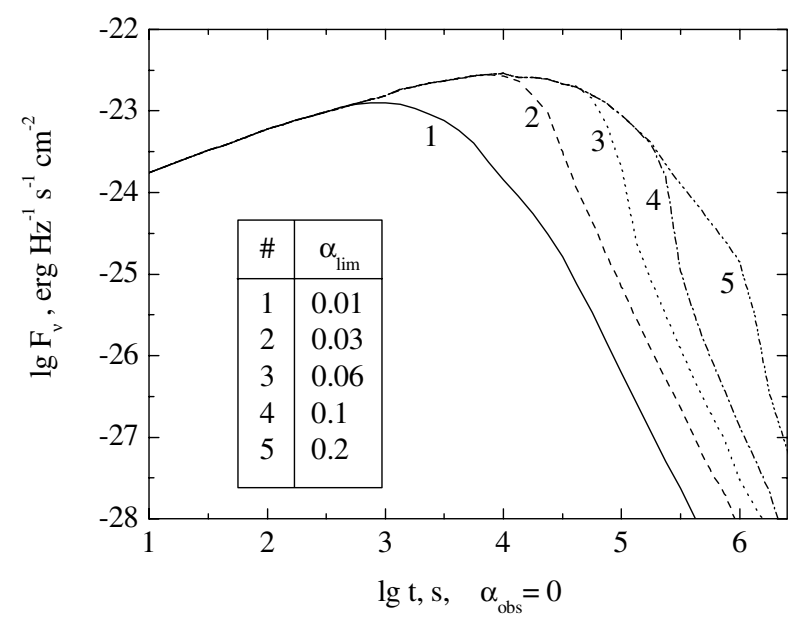

Fig. 4. Afterglow light curves at frequency $v=5 \times 10^{14} \mathrm{~Hz}$ and the observational angle $\alpha_{\text {obs }}=0$ for different values of the limiting angle $\alpha_{\text {lim }}$.

Now, if we fix the time of the observation by the value, say, $t_{\mathrm{obs}}^{\prime}=10^{4} c$, there is no reason to increase the value of the limiting angle in our jet structure to more than $\alpha_{\text {lim }}^{\prime}=0.037$ (Fig. 2) in the calculation of spectra, because this does not produce any effect on the resulting spectra and they appear as if they were produced by a non-limited structure.

We present the calculated spectra and light curves at zero observational angle and at different values of the limiting angle in Figs. 3 and 4.

The changed form of the spectra has two peak fluxes at some values of the limiting angle and is the consequence of the ring intensity on the radiative surface (Tolstov \& Blinnikov 2003).

If we look at the ring structure we can see that the higher the light frequency, the closer the maximum of brightness is to the edge of the image. The flux from the observed image can be calculated by the formula

$$
F_{v}=2 \pi \int_{\cos \theta_{\max }}^{1} I(\cos \theta, r, v) \cos \theta \mathrm{d} \cos \theta
$$




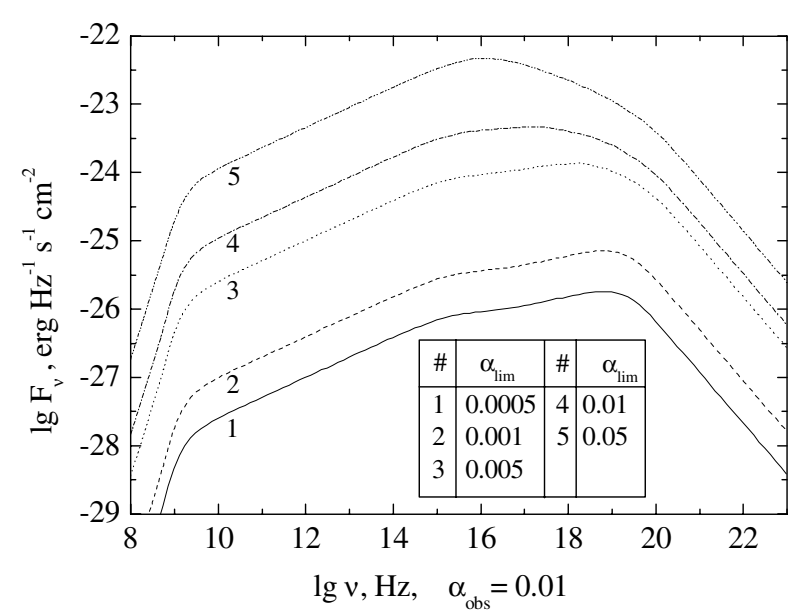

Fig. 5. Instantaneous afterglow spectra at time $t=10^{4} \mathrm{~s}$ and the observational angle $\alpha_{\mathrm{obs}}=0.01$ for different values of the limiting angle $\alpha_{\lim }$.

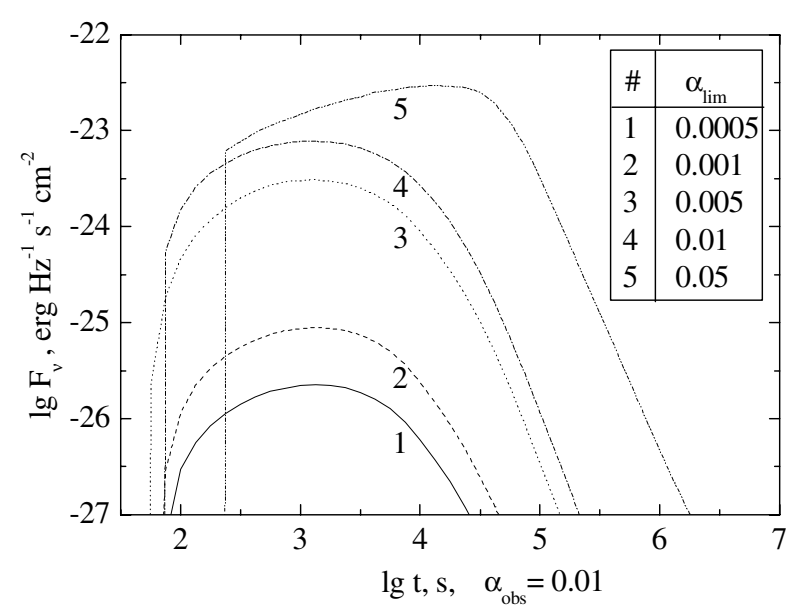

Fig. 6. Afterglow light curves at the frequency $v=5 \times 10^{14} \mathrm{~Hz}$ and the observational angle $\alpha_{\text {obs }}=0.01$ for different values of the limiting angle $\alpha_{\text {lim. }}$.

where $\theta$ is the angle between the point on the radiating surface and the center of the structure (see Fig. 1), $\theta_{\max }$ corresponds to the edge of the image. In case of no limiting angle the flux monotonically decreases at the right part of the spectrum as long as the light frequency increases. With a limiting angle some maxima of the intensity at low frequencies are excluded from the flux integral and the flux value at high frequencies is comparable to the one at low frequencies.

The light curves do not show "jet breaks" due to the limiting angle. This results from the decrease of the radiation arriving at the observer from the shock limited by $\alpha_{\text {lim. }}$. The larger the value of $\alpha_{\text {lim }}$, the less the radiation at some frequencies towards the observer. A few days after the burst at $\alpha_{\text {lim }}=0.2$ we can see the break typical of some observed optical afterglows (Zhang \& Meszaros 2003).

In Figs. 5 and 6 we present the calculated spectra and light curves at the observational angle $\alpha_{\text {obs }}=0.01$. At small values of the limiting angles we can also see changes in the spectra while for the light curves we did not have this effect. Some of the presented light curves are cut at early moments. This is

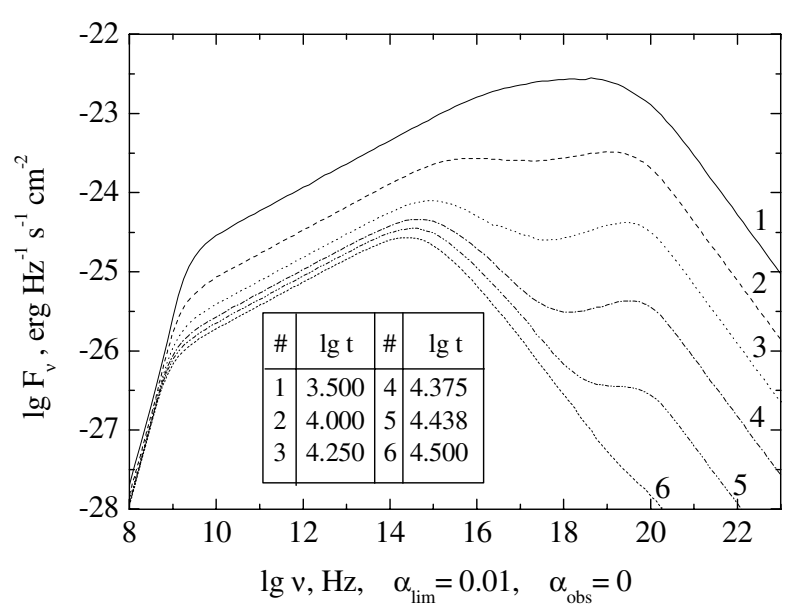

Fig. 7. Instantaneous afterglow spectra at the limiting angle $\alpha_{\mathrm{obs}}=$ 0.01 and the observational angle $\alpha_{\mathrm{obs}}=0$ for different values of time $t$.

the consequence of solving the transfer equation only up to the Lorentz factor value $\gamma=1000$. At larger values of the Lorentz factor we suppose that the matter is not radiative and while $\alpha_{\text {obs }}=0$ this effect is not seen, at $\alpha_{\text {obs }}=0.01$ some of the characteristics appear at a Lorentz factor value $\gamma \geq 1000$.

In Fig. 7 we show the calculated spectra for different values of time at fixed limiting and observational angles, which show the evolution of the peculiarities described above and can be used for comparison with the real afterglow spectra if the volume of observational data increases.

\section{Conclusions}

It is widely believed that GRBs are born in jet geometry. In this case the resulting afterglow radiation becomes highly collimated. The numerical calculations of light curves in these models (Granot \& Kumar 2003; Salmonson 2003) are based on some assumptions on intensity at the propagating shock front. The shape of the local spectral emissivity is usually approximated as a broken power-law with some typical breaks corresponding to synchrotron radiation. As we can see from our results the spectra can have some peculiarities and shapes that are different from a power law both in a head-on jet (Fig. 3) and at some angle to the jet axis (Fig. 5).

Of course, our consideration does not take into account some effects of the jet model and the exact numerical calculations should be at least two-dimensional to allow for lateral expansion and the angular structure of the jet.

Nevertheless, the accurate calculation of intensity, using a relativistic transfer equation, can have an influence on the shape of spectra and light curves of GRB afterglows. Constructing a more precise model using exact numerical calculations can help to explain the peculiarities of GRB afterglow and may shed some light on the nature of the GRB phenomenon.

Acknowledgements. I am grateful to S. I. Blinnikov for posing the problem and for valuable discussions. Also I wish to convey my sincerest thanks to H. Spruit for the kind hospitality at the Max-Plank Institute for Astrophysics, under the auspices of which this work was performed. The work in Russia is partly supported by RBRF grant 02-02-16500. 


\section{References}

Blandford, R. D., \& McKee, C. F. 1976, Phys. Fluids, 19, 1130 Bianco, C. L., \& Ruffini, R. 2004 [arXiv:astro-ph/0403379] Canizzo, J. K., Gehrels, N., \& Vishniac, E. T. 2003 [arXiv: astro-ph/0310113]

Covino, S., Lazzati, D., Ghisellini, G., et al. 1999, A\&A, 348, L1 Granot, J., \& Sari, R. 2001, ApJ, 568, 820

Granot, J., Panaitescu, A., Kumar, P., \& Woosley, S. E. 2002, ApJ, 570, L61

Granot, J., \& Kumar, P. 2003, ApJ, 591, 1086

Harrison, F. A., Bloom, J. S., Frail, D. A., et al. 1999, ApJ, 121, 523

Kulkarni, S. R., Djorgovski, S. G., Odewhan, S. C., et al. 1999, Nature, 389, 398

Kumar, P., \& Panaitescu, A. 2000, ApJ, 541, L9
Panaitescu, A., \& Meszaros, P. 1999, ApJ, 526, 707

Rybicki, G. B., \& Lightman, A. P. 1979, Radiative processes in astrophysics (New York: Wiley)

Salmonson, J. D. 2003, ApJ, 592, 1002

Sari, R., Piran, T., \& Halpern, G. P. 1999a, ApJ, 519, L17

Sari, R. 1999b, ApJ, 524, L43

Sari, R., Piran, T., \& Narayan, R. 1999c, ApJ, 489, L33

Stanek, K. Z., Garnavich, P. M., Kaluznyj, J., et al. 1999, ApJ, 522, L39

Tolstov, A. G., \& Blinnikov, S. I. 2003, AstL, 29, 403

Wei, D. M., \& Jin, Z. P. 2003, A\&A, 400, 415

Wijers, R. A. M. J., Vreeswijk, P. M., Galama, T. J., et al. 1999, ApJ, 523, L33

Zhang, B., \& Meszaros, P. 2003 [arXiv: astro-ph/0311321] 